FedUni ResearchOnline

https://researchonline.federation.edu.au

This is the author's accepted version of the following publication:

Kruger, A. (2006) About regularity of collections of sets. Set-Valued Analysis, 14(2), 187-206.

The version displayed here may differ from the final published version.

The final publication is available at:

http://dx.doi.org/10.1007/s11228-006-0014-8

Copyright $@ 2006$, Springer. 


\title{
About Regularity of Collections of Sets
}

Received: date / Accepted: date

\begin{abstract}
The paper continues investigations of stationarity and regularity properties of collections of sets in normed spaces. It contains a summary of different characterizations (both primal and dual) of regularity and a list of sufficient conditions for a collection of sets to be regular.
\end{abstract}

Keywords variational analysis, normal cone, optimality, extremality, stationarity, regularity, set-valued mapping, Asplund space

Mathematics Subject Classification (2000) 90C46, 90C48, 49K27; Secondary: $58 \mathrm{C} 20,58 \mathrm{E} 30$

\section{Introduction}

This paper continues investigations of stationarity and regularity properties of collections of sets in normed spaces started in [19]. See [19] for motivations as well as comparisons of different approaches.

Starting with the pioneering work by Dubovitskii and Milyutin [10] it is quite natural when dealing with optimality conditions to reformulate optimality in the original optimization problem as a (some kind of) extremal behaviour of a certain collection of sets. An easy example is a problem of unconditional minimization of a real-valued function $\varphi: X \rightarrow R$. If $x^{\circ} \in X$ one can consider the sets $\Omega_{1}=$ epi $\varphi=$ $\{(x, \mu) \in X \times R: \varphi(x) \leq \mu\}$ (the epigraph of $\varphi$ ) and $\Omega_{2}=X \times\left\{\mu: \mu \leq \varphi\left(x^{\circ}\right)\right\}$ (the lower halfspace). The local optimality of $x^{\circ}$ is then equivalent to the condition $\Omega_{1} \cap$ int $\Omega_{2} \cap\left(x^{\circ}+\rho B\right)=\emptyset$ for some $\rho>0$.

The concept of extremality for a collection of sets was first defined in [21]. This definition was extended to stationarity in [14-16], where two definitions of

School of Information Technology and Mathematical Sciences, Centre of Information and Applied Optimization, University of Ballarat, POB 663, Ballarat, Vic 3350, Australia

E-mail: a.kruger@ballarat.edu.au 
stationarity (one of them was at first called "extended extremality") were introduced. The relation between extremality and stationarity for collections of sets is similar to that between optimality and stationarity for optimization problems.

Regularity can be considered as the property opposite to stationarity (see [19]). Regularity properties of collections of sets play an important role in different fields of optimization and approximation: constraint qualifications, error bounds, convergence analysis, etc. (see $[3,7,26]$ for numerous examples). They are also closely related to similar properties of multifunctions and can be used in analysis, in particular, in nonsmooth calculus.

Following [16,18], extremality-stationarity-regularity properties are defined in the paper with the help of some constants providing quantitative estimates of the corresponding properties. A similar approach (for different properties) is undertaken in the recent paper [2].

Examining and comparing different regularity concepts for collections of sets has attracted recently considerable attention in the literature (see $[2-5,7,19,26$, 27]), although according to [2] some traces of such considerations can be found in the 1940 paper by M. Krein.

The strong regularity concept investigated in the current paper is closely related to the metric regularity property (sometimes referred to as pseudo regularity) of multifunctions. Different characterizations of strong regularity of collections of sets are presented as well as some relations to other properties. The main emphasis in the current paper is on primal space conditions.

The definitions of the constants, the relations between them and the corresponding stationary and regularity concepts developed in the current paper are very similar to those for nonsmooth functions and multifunctions (see [18-20]). Actually these are different applications of the same variational approach.

In the convex case there exists another set of definitions of regularity properties (based on linear regularity [3]) with numerous interesting equivalences and other relations (see $[3,7,26])$. Linear regularity is defined as a global property. However, local versions of this property can also be of interest. Note that (local) linear regularity is in general weaker than the strong regularity property considered here (see the example at the end of subsection 3.3). Thus, two sets of regularity conditions exist in parallel with many similarities between them.

The paper is organized as follows. The definitions of extremality, stationarity and regularity for the collection of sets are introduced in Section 2. Some primal space constants characterizing the mutual arrangement of sets in space are used in the definitions. Section 3 contains a summary of different characterizations (both primal and dual) of strong regularity with relations to other properties: metric inequality, error bounds, weak sharp minima, Jameson's property $(G)$, strong additive regularity. Some more constants for quantitative characterization of the corresponding properties are introduced. The final Section 4 is devoted to considering examples of strongly regular collections of sets. It contains a list of sufficient conditions for a collection of sets to be strongly regular.

Mainly standard notations are used throughout the paper. A closed unit ball in a normed space is denoted by $B$. If $\Omega$ is a set then $\operatorname{int} \Omega, \operatorname{bd} \Omega$ and $\operatorname{cl} \Omega$ are respectively its interior, the boundary and the closure. When considering product spaces we will always assume that they are equipped with the maximum-type norm: $\left\|\left(x_{1}, x_{2}\right)\right\|=\max \left(\left\|x_{1}\right\|,\left\|x_{2}\right\|\right)$. 


\section{Extremality, stationarity and regularity}

Let us consider a collection of sets $\Omega_{1}, \Omega_{2}, \ldots, \Omega_{n}(n>1)$ in a normed space $X$ with $x^{\circ} \in \cap_{i=1}^{n} \Omega_{i}$.

The following constant can be used for characterizing the mutual arrangement of the sets $\Omega_{1}, \Omega_{2}, \ldots, \Omega_{n}$ near $x^{\circ}([18,19])$ :

$$
\begin{aligned}
\theta_{\rho}\left[\Omega_{1}, \ldots, \Omega_{n}\right]\left(x^{\circ}\right)=\sup \{r & \geq 0: \\
& \left.\left(\bigcap_{i=1}^{n}\left(\Omega_{i}-a_{i}\right)\right) \bigcap\left(x^{\circ}+\rho B\right) \neq \emptyset, \forall a_{i} \in r B\right\} .
\end{aligned}
$$

It shows how far the sets can be "pushed apart" while still intersecting in a neighborhood of $x^{\circ}$. Evidently $\theta_{\rho}\left[\Omega_{1}, \ldots, \Omega_{n}\right]\left(x^{\circ}\right)$ is nonnegative (and can be equal to $+\infty$ ) and nondecreasing as a function of $\rho$. Moreover,

$$
\lim _{\rho \rightarrow+0} \theta_{\rho}\left[\Omega_{1}, \ldots, \Omega_{n}\right]\left(x^{\circ}\right)=0
$$

unless $x^{\circ} \in$ int $\cap_{i=1}^{n} \Omega_{i}$ ([19], Proposition 3).

A slightly more general form of (1) can be of interest:

$$
\begin{aligned}
\theta_{\rho}\left[\Omega_{1}, \ldots, \Omega_{n}\right]\left(\omega_{1}, \ldots, \omega_{n}\right)= & \sup \{r \geq 0: \\
& \left.\left(\bigcap_{i=1}^{n}\left(\Omega_{i}-\omega_{i}-a_{i}\right)\right) \bigcap(\rho B) \neq \emptyset, \forall a_{i} \in r B\right\} .
\end{aligned}
$$

This constant corresponds to the case when instead of the common point $x^{\circ} \in \cap_{i=1}^{n} \Omega_{i}$ each of the sets $\Omega_{i}$ is considered near its own point $\omega_{i} \in \Omega_{i}$, $i=1,2, \ldots, n$. The sets do not need to be intersecting. It is equivalent to considering the collection of translated sets $\Omega_{1}-\omega_{1}, \Omega_{2}-\omega_{2}, \ldots, \Omega_{n}-\omega_{n}$ near 0:

$$
\theta_{\rho}\left[\Omega_{1}, \ldots, \Omega_{n}\right]\left(\omega_{1}, \ldots, \omega_{n}\right)=\theta_{\rho}\left[\Omega_{1}-\omega_{1}, \ldots, \Omega_{n}-\omega_{n}\right](0) .
$$

If $\omega_{1}=\omega_{2}=\ldots=\omega_{n}=x^{\circ}$ then, of course,

$$
\theta_{\rho}\left[\Omega_{1}, \ldots, \Omega_{n}\right]\left(\omega_{1}, \ldots, \omega_{n}\right)=\theta_{\rho}\left[\Omega_{1}, \ldots, \Omega_{n}\right]\left(x^{\circ}\right) .
$$

When investigating stationarity-regularity properties it is important to know how fast $\theta_{\rho}\left[\Omega_{1}, \ldots, \Omega_{n}\right]\left(x^{\circ}\right)$ and $\theta_{\rho}\left[\Omega_{1}, \ldots, \Omega_{n}\right]\left(\omega_{1}, \ldots, \omega_{n}\right)$ approach 0 in comparison with $\rho$. This can be characterized by the following "linearized" constants:

$$
\begin{aligned}
\theta\left[\Omega_{1}, \ldots, \Omega_{n}\right]\left(x^{\circ}\right) & =\liminf _{\rho \rightarrow+0} \frac{\theta_{\rho}\left[\Omega_{1}, \ldots, \Omega_{n}\right]\left(x^{\circ}\right)}{\rho} . \\
\theta\left[\Omega_{1}, \ldots, \Omega_{n}\right]\left(\omega_{1}, \ldots, \omega_{n}\right) & =\liminf _{\rho \rightarrow+0} \frac{\theta_{\rho}\left[\Omega_{1}, \ldots, \Omega_{n}\right]\left(\omega_{1}, \ldots, \omega_{n}\right)}{\rho} .
\end{aligned}
$$

The last step in the process of defining the collection of constants characterizing local properties of the collection of sets $\Omega_{1}, \Omega_{2}, \ldots, \Omega_{n}$ near $x^{\circ}$ is to consider 
the limit in the right-hand side of (4) when the points $\omega_{1}, \omega_{2}, \ldots, \omega_{n}$ are not fixed, but approach $x^{\circ}$. We arrive at the next constant:

$$
\hat{\theta}\left[\Omega_{1}, \ldots, \Omega_{n}\right]\left(x^{\circ}\right)=\liminf _{\substack{\Omega_{\Omega_{i}} \\ \omega_{i} x^{\circ} \\ \rho \rightarrow+0}} \frac{\theta_{\rho}\left[\Omega_{1}, \ldots, \Omega_{n}\right]\left(\omega_{1}, \ldots, \omega_{n}\right)}{\rho}
$$

The notation $\omega \stackrel{\Omega}{\rightarrow} x$ in (5) means that $\omega \rightarrow x$ with $\omega \in \Omega$.

Proposition 1 The following inequality holds true:

$$
\hat{\theta}\left[\Omega_{1}, \ldots, \Omega_{n}\right]\left(x^{\circ}\right) \leq \liminf _{\omega_{i} \rightarrow x_{i}^{\circ}} \theta\left[\Omega_{1}, \ldots, \Omega_{n}\right]\left(\omega_{1}, \ldots, \omega_{n}\right) .
$$

Proof Denote for brevity

$$
\psi_{\rho}\left(\omega_{1}, \ldots, \omega_{n}\right)=\frac{\theta_{\rho}\left[\Omega_{1}, \ldots, \Omega_{n}\right]\left(\omega_{1}, \ldots, \omega_{n}\right)}{\rho} .
$$

By the definition of the lower limit one can write

$$
\begin{aligned}
\hat{\theta}\left[\Omega_{1}, \ldots, \Omega_{n}\right]\left(x^{\circ}\right)=\lim _{\delta \rightarrow+0} \inf _{\substack{\omega_{i} \in \Omega_{i} \cap\left(x^{\circ}+\delta B\right) \\
0<\rho \leq \delta}} \psi_{\rho}\left(\omega_{1}, \ldots, \omega_{n}\right)= \\
\quad \lim _{\delta \rightarrow+0} \inf _{\omega_{i} \in \Omega_{i} \cap\left(x^{\circ}+\delta B\right)} \inf _{0<\rho \leq \delta} \psi_{\rho}\left(\omega_{1}, \ldots, \omega_{n}\right) .
\end{aligned}
$$

The legality of the replacement of the "double" infimum in the above formula by two separate ones is quite obvious. For any $\omega_{i} \in \Omega_{i}, i=1,2, \ldots, n$, and any $0<\delta^{\prime} \leq \delta$ one has

$$
\inf _{0<\rho \leq \delta} \psi_{\rho}\left(\omega_{1}, \ldots, \omega_{n}\right) \leq \inf _{0<\rho \leq \delta^{\prime}} \psi_{\rho}\left(\omega_{1}, \ldots, \omega_{n}\right) .
$$

Consequently,

$$
\begin{aligned}
\inf _{0<\rho \leq \delta} \psi_{\rho}\left(\omega_{1}, \ldots, \omega_{n}\right) & \leq \lim _{\delta^{\prime} \rightarrow 0} \inf _{0<\rho \leq \delta^{\prime}} \psi_{\rho}\left(\omega_{1}, \ldots, \omega_{n}\right)= \\
& \liminf _{\rho \rightarrow+0} \psi_{\rho}\left(\omega_{1}, \ldots, \omega_{n}\right)=\theta\left[\Omega_{1}, \ldots, \Omega_{n}\right]\left(\omega_{1}, \ldots, \omega_{n}\right) .
\end{aligned}
$$

(6) follows from (7) and (8).

Remark 1 The above proof is valid for the "combined" lower limit of any function of several variables.

Inequality (6) can be strict.

Example 1 Consider two sets in $\mathbb{R}^{2}: \Omega_{1}=\left\{(x, y) \in \mathbb{R}^{2}: \varphi(x) \leq y\right\}$ and $\Omega_{2}=$ $\left\{(x, y) \in \mathbb{R}^{2}: y \leq 0\right\}$, where the function $\varphi: \mathbb{R} \rightarrow \mathbb{R}$ is defined in the following way: $\varphi(x)=x$ if $x \leq 0, \varphi(x)=x-1 / n$ if $1 / n<x \leq 1 /(n-1), n=2,3, \ldots$, $\varphi(x)=x-1 / 2$ if $x>1 / 2$.

Take $\omega_{1}=\left(x_{1}, y_{1}\right) \in \Omega_{1}$ and $\omega_{2}=\left(x_{2}, y_{2}\right) \in \Omega_{2}$. If $\varphi\left(x_{1}\right)<y_{1}$ or $y_{2}<0$ then $\theta_{\rho}\left[\Omega_{1}, \Omega_{2}\right]\left(\omega_{1}, \omega_{2}\right) \geq y_{1}-\varphi\left(x_{1}\right)-y_{2}>0$ for any $\rho>0$ and consequently 
$\theta\left[\Omega_{1}, \Omega_{2}\right]\left(\omega_{1}, \omega_{2}\right)=\infty$. If $\varphi\left(x_{1}\right)=y_{1}$ and $y_{2}=0$ then $\theta\left[\Omega_{1}, \Omega_{2}\right]\left(\omega_{1}, \omega_{2}\right)=1$. Thus, $\theta\left[\Omega_{1}, \Omega_{2}\right]\left(\omega_{1}, \omega_{2}\right) \geq 1$ for any $\omega_{1} \in \Omega_{1}$ and $\omega_{2} \in \Omega_{2}$.

On the other hand, take $x_{n}=1 / n+1 / n^{2}, y_{n}=1 / n^{2}, \rho_{n}=1 / n$. Then $\omega_{1 n}=$ $\left(x_{n}, y_{n}\right) \in \Omega_{1}, n=1,2, \ldots, \omega_{2}=0 \in \Omega_{2}$ and $\theta_{\rho_{n}}\left[\Omega_{1}, \Omega_{2}\right]\left(\omega_{1 n}, \omega_{2}\right) \leq 1 / n^{2}$. Obviously, $\omega_{1 n} \rightarrow 0, \theta_{\rho_{n}}\left[\Omega_{1}, \Omega_{2}\right]\left(\omega_{1 n}, \omega_{2}\right) / \rho_{n} \rightarrow 0$ and consequently $\hat{\theta}\left[\Omega_{1}, \Omega_{2}\right](0)=0$.

The constants (3)-(5) are in a sense derivative-like objects. (3) and (4) can be considered as analogs of the usual derivative, while (5) has some properties of the strict derivative: it accumulates information about local properties of the sets not only at a given point but also at all nearby points.

All the constants (1)-(5) are nonnegative. When investigating extremality-stationarity-regularity properties of the collection of sets one needs to check whether the corresponding constant is zero or strictly positive.

Evidently

$$
0 \leq \hat{\theta}\left[\Omega_{1}, \ldots, \Omega_{n}\right]\left(x^{\circ}\right) \leq \theta\left[\Omega_{1}, \ldots, \Omega_{n}\right]\left(x^{\circ}\right) \leq+\infty
$$

Definition 1 The collection of sets $\Omega_{1}, \Omega_{2}, \ldots, \Omega_{n}$ is

(i) extremal at $x^{\circ}$ if $\theta_{\rho}\left[\Omega_{1}, \ldots, \Omega_{n}\right]\left(x^{\circ}\right)=0$ for all $\rho>0$.

(ii) locally extremal at $x^{\circ}$ if $\theta_{\rho}\left[\Omega_{1}, \ldots, \Omega_{n}\right]\left(x^{\circ}\right)=0$ for some $\rho>0$.

(iii) stationary at $x^{\circ}$ if $\theta\left[\Omega_{1}, \ldots, \Omega_{n}\right]\left(x^{\circ}\right)=0$.

(iv) weakly stationary at $x^{\circ}$ if $\hat{\theta}\left[\Omega_{1}, \ldots, \Omega_{n}\right]\left(x^{\circ}\right)=0$.

(v) regular at $x^{\circ}$ if $\theta\left[\Omega_{1}, \ldots, \Omega_{n}\right]\left(x^{\circ}\right)>0$.

(vi) strongly regular at $x^{\circ}$ if $\hat{\theta}\left[\Omega_{1}, \ldots, \Omega_{n}\right]\left(x^{\circ}\right)>0$.

The extremality of the collection of sets was introduced (in a different way) in [21], where dual necessary conditions in the form of the generalized Euler equation were formulated. This result currently known as the extremal principle has been applied to investigating different optimization problems (see [22,23]). Stationarity and regularity properties were considered in [19]. The first version of the weak stationarity was defined (under a different name) in [14] (see also [16, 17]).

The weak stationarity appears to be a natural extension of the extremality property in the sense that the dual necessary conditions remain valid for it (in Asplund spaces) and become also sufficient (the extended extremal principle $[14,18]$ ).

The (strong) regularity of the collection of sets is a natural counterpart of the (weak) stationarity property and can be used e.g. when formulating constraint qualifications in mathematical programming. The strong regularity is closely related to the metric regularity of multifunctions [11,12]. This will be the main object of investigation in the current paper.

\section{Characterizations of strong regularity}

This section contains the summary of different characterizations of strong regularity based on [19]. 
3.1 Equivalent definition of strong regularity

The next proposition is an immediate consequence of (5).

Proposition 2 The collection of sets $\Omega_{1}, \Omega_{2}, \ldots, \Omega_{n}$ is strongly regular at $x^{\circ}$ if and only if the following condition holds:

(a) There exists an $\alpha>0$ and $a \delta>0$ such that

$$
\left(\bigcap_{i=1}^{n}\left(\Omega_{i}-\omega_{i}-a_{i}\right)\right) \bigcap(\rho B) \neq \emptyset
$$

for all $\rho \in(0, \delta], \omega_{i} \in \Omega_{i} \cap\left(x^{\circ}+\delta B\right), a_{i} \in \alpha \rho B, i=1,2, \ldots, n$.

$\hat{\theta}\left[\Omega_{1}, \ldots, \Omega_{n}\right]\left(x^{\circ}\right)$ equals to the exact upper bound of all such $\alpha$.

Corollary 1 The collection of sets $\Omega_{1}, \Omega_{2}, \ldots, \Omega_{n}$ is strongly regular at $x^{\circ}$ provided that $x^{\circ} \in$ int $\cap_{i=1}^{n} \Omega_{i}$. In this case $\hat{\theta}\left[\Omega_{1}, \ldots, \Omega_{n}\right]\left(x^{\circ}\right)=+\infty$.

Corollary 1 implies that only the case $x^{\circ} \in \mathrm{bd} \cap_{i=1}^{n} \Omega_{i}$ is of interest from the point of view of investigating the strong regularity property of collections of sets. It is not difficult to show ([19], Proposition 8) that $\hat{\theta}\left[\Omega_{1}, \ldots, \Omega_{n}\right]\left(x^{\circ}\right) \leq 1$ in this case.

\subsection{Case $n=2$}

If $\omega_{1} \in \Omega_{1}, \omega_{2} \in \Omega_{2}$ the next constant can be used instead of (2):

$$
\begin{aligned}
\theta_{\rho}^{\prime}\left[\Omega_{1}, \Omega_{2}\right]\left(\omega_{1}, \omega_{2}\right)= & \sup \{r \geq 0: \\
& \left.r B \subset\left(\left(\Omega_{1}-\omega_{1}\right) \cap(\rho B)\right)-\left(\left(\Omega_{2}-\omega_{2}\right) \cap(\rho B)\right)\right\} .
\end{aligned}
$$

Similar to (4), (5) the following two constants are defined based on (10):

$$
\begin{gathered}
\theta^{\prime}\left[\Omega_{1}, \Omega_{2}\right]\left(\omega_{1}, \omega_{2}\right)=\liminf _{\rho \rightarrow+0} \frac{\theta_{\rho}^{\prime}\left[\Omega_{1}, \Omega_{2}\right]\left(\omega_{1}, \omega_{2}\right)}{\rho}, \\
\hat{\theta}^{\prime}\left[\Omega_{1}, \Omega_{2}\right]\left(x^{\circ}\right)=\liminf _{\substack{\Omega_{1} \\
\omega_{1} \stackrel{\Omega_{1}, \alpha_{2}}{\rightarrow} \rightarrow x^{\circ} \\
\rho \rightarrow+0}} \frac{\theta_{\rho}^{\prime}\left[\Omega_{1}, \Omega_{2}\right]\left(\omega_{1}, \omega_{2}\right)}{\rho} .
\end{gathered}
$$
(12).

The next proposition follows from [19], Proposition 18, and definitions (10)-

Proposition 3 The following assertions are equivalent:

(i) The collection of sets $\Omega_{1}, \Omega_{2}$ is strongly regular at $x^{\circ}$.

(ii) $\hat{\theta}^{\prime}\left[\Omega_{1}, \Omega_{2}\right]\left(x^{\circ}\right)>0$.

(iii) There exists an $\alpha>0$ and $a \delta>0$ such that

$$
\alpha \rho B \subset\left(\left(\Omega_{1}-\omega_{1}\right) \cap(\rho B)\right)-\left(\left(\Omega_{2}-\omega_{2}\right) \cap(\rho B)\right)
$$

for all $\rho \in(0, \delta], \omega_{1} \in \Omega_{1} \cap\left(x^{\circ}+\delta B\right), \omega_{2} \in \Omega_{2} \cap\left(x^{\circ}+\delta B\right)$. 
The general case $n \geq 2$ can be easily reduced to considering a collection of two sets.

Proposition 4 The collection of sets $\Omega_{1}, \Omega_{2}, \ldots, \Omega_{n}$ is strongly regular at $x^{\circ}$ if and only if the collection of two sets $\tilde{\Omega}_{1}=\Omega_{1} \times \Omega_{2} \times \ldots \times \Omega_{n}, \tilde{\Omega}_{2}=$ $\{(x, x, \ldots, x): x \in X\}$ in $X^{n}$ is strongly regular at $\tilde{x}^{\circ}=\left(x^{\circ}, x^{\circ}, \ldots, x^{\circ}\right) \in \tilde{\Omega}_{1} \cap \tilde{\Omega}_{2}$.

\subsection{Strong metric inequality}

Strong regularity of the collection of sets can be determined by comparing some point-to-set distances.

Let $d(\cdot, \cdot)$ be the distance function in $X$ associated with the norm. We will keep the same notation for point-to-set distances. Thus, $d(x, \Omega)=\inf _{\omega \in \Omega}\|x-\omega\|$ is the distance from a point $x$ to a set $\Omega$ and $d(x, \emptyset)=\infty$. The following constant can be used for characterizing the regularity property of the collection of sets $\Omega_{1}, \Omega_{2}$, $\ldots, \Omega_{n}$ :

$$
\hat{\vartheta}\left[\Omega_{1}, \ldots, \Omega_{n}\right]\left(x^{\circ}\right)=\limsup _{\substack{x \rightarrow x^{\circ} \\ x_{i} \rightarrow 0}}\left(d\left(x, \bigcap_{i=1}^{n}\left(\Omega_{i}-x_{i}\right)\right) / \max _{1 \leq i \leq n} d\left(x+x_{i}, \Omega_{i}\right)\right)_{。} .
$$

The "extended" division operation $(\cdot / \cdot)_{\circ}$ is used in (13) to simplify the definition. It legalizes division by zero. The rules are as follows:
1) $(\alpha / \beta)_{\circ}=\alpha / \beta$, if $\beta \neq 0$;
2) $(\alpha / 0)_{\circ}=+\infty$, if $\alpha>0$;
3) $(\alpha / 0)_{\circ}=-\infty$, if $\alpha<0$;
4) $(0 / 0)_{\circ}=0$.

The fourth rule is the most important one here. In the case $x^{\circ} \in$ int $\cap_{i=1}^{n} \Omega_{i}$ it automatically leads to $\hat{\vartheta}\left[\Omega_{1}, \ldots, \Omega_{n}\right]\left(x^{\circ}\right)=0$. Otherwise the points where the numerator and the denominator are both zero in the right-hand side of (13) can be ignored when calculating the limit.

\section{Theorem 1 ([19])}

(i) $\hat{\vartheta}\left[\Omega_{1}, \ldots, \Omega_{n}\right]\left(x^{\circ}\right)=1 / \hat{\theta}\left[\Omega_{1}, \ldots, \Omega_{n}\right]\left(x^{\circ}\right)$.

(ii) The collection of sets $\Omega_{1}, \Omega_{2}, \ldots, \Omega_{n}$ is strongly regular at $x^{\circ}$ if and only if there exists $a \beta>0$ and $a \delta>0$ such that the strong metric inequality holds:

$$
d\left(x, \bigcap_{i=1}^{n}\left(\Omega_{i}-x_{i}\right)\right) \leq \beta \max _{1 \leq i \leq n} d\left(x+x_{i}, \Omega_{i}\right)
$$

for all $x \in x^{\circ}+\delta B, x_{i} \in \delta B, i=1,2, \ldots, n$.

$\hat{\vartheta}\left[\Omega_{1}, \ldots, \Omega_{n}\right]\left(x^{\circ}\right)$ equals to the exact lower bound of all such $\beta$. 27]

The condition formulated in Proposition 1 implies the metric inequality [11,

$$
d\left(x, \bigcap_{i=1}^{n} \Omega_{i}\right) \leq \beta \max _{1 \leq i \leq n} d\left(x, \Omega_{i}\right), \quad \forall x \in x^{\circ}+\delta B .
$$


If (15) is valid for all $\delta>0$ then the collection of sets is said to be boundedly linear regular [3], or simply linear regular $[3,26]$ if $(15)$ holds with $\delta=\infty$. Both properties are important when investigating convex optimization problems. One can consider some other regularity properties of collections of sets with interesting relations to linear regularity (see [26]). Note that due to Proposition 1 these properties are in a sense weaker than the strong regularity concept considered here.

(14) is certainly stronger than (15) even in the convex case. Take for instance $\Omega_{1}=\Omega_{2}=\left\{(x, y) \in \mathbb{R}^{2}: y=0\right\}$. Then (15) holds true (with $\beta=1, \delta=\infty$ ) while (14) does not.

\subsection{Error bounds}

The regularity concepts discussed above are related to error bounds in mathematical programming (see $[3,26]$ ).

Let $f=\left(f_{1}, f_{2}, \ldots, f_{n}\right)$, where $f_{i}: X \rightarrow \overline{\mathbb{R}}, i=1,2, \ldots, n$. For each $i$ define $S_{f_{i}}=\left\{x \in X: f_{i}(x) \leq 0\right\}$, and $S_{f}=\cap_{i=1}^{n} S_{f_{i}}$. Let us start with the local version of the (slightly modified) corresponding definition from [26].

Definition $2 f$ has an error bound at $x^{\circ} \in S_{f}$ if there exists a $\beta>0$ and a $\delta>0$ such that

$$
d\left(x, S_{f}\right) \leq \beta \max _{i=1, \ldots, n}\left[f_{i}(x)\right]_{+}
$$

for all $x \in x^{\circ}+\delta B$.

The notation $[\alpha]_{+}=\max (\alpha, 0)$ is used in (16).

We will need a stronger version of Definition 2.

Definition $3 f$ has a strong error bound at $x^{\circ} \in S_{f}$ if there exists a $\beta>0$ and a $\delta>0$ such that

$$
d\left(x, \bigcap_{i=1}^{n}\left(S_{f_{i}}-x_{i}\right)\right) \leq \beta \max _{i=1, \ldots, n}\left[f_{i}\left(x+x_{i}\right)\right]_{+}
$$

for all $x \in x^{\circ}+\delta B, x_{i} \in \delta B, i=1,2, \ldots, n$.

For the collection of sets $\Omega_{1}, \Omega_{2}, \ldots, \Omega_{n}$ one can define

$$
f_{i}(x)=d\left(x, \Omega_{i}\right), i=1,2, \ldots, n .
$$

Then $\Omega_{i}=S_{f_{i}}, \cap_{i=1}^{n} \Omega_{i}=S_{f}$ and (15) takes the form (16), while (14) takes the form (17). Thus, the (strong) metric inequality is equivalent to $f=\left(f_{1}, f_{2}, \ldots, f_{n}\right)$ with the components defined by (18) having a (strong) error bound.

Part (ii) of Proposition 1 implies the following statement.

Proposition 5 The collection of sets $\Omega_{1}, \Omega_{2}, \ldots, \Omega_{n}$ is strongly regular at $x^{\circ}$ if and only if $f=\left(f_{1}, f_{2}, \ldots, f_{n}\right)$ (with the components defined by (18)) has a strong error bound at $x^{\circ}$.

$\hat{\vartheta}\left[\Omega_{1}, \ldots, \Omega_{n}\right]\left(x^{\circ}\right)$ equals to the exact lower bound of all $\beta$ from Definition 3. 
3.5 Weak sharp minima

Similar to error bounds a close optimization theory concept of weak sharp minima (see $[6-8,29])$ can be related to the regularity of collections of sets.

Let $f: X \rightarrow \overline{\mathbb{R}}, x^{\circ} \in X$. Define $S_{f}\left(x^{\circ}\right)=\left\{x \in X: f(x) \leq f\left(x^{\circ}\right)\right\}$. We will again start with the local version of the (slightly modified) corresponding definition from [6]. Note that it differs also from the definition of the local weak sharp minima as it is formulated in [6].

Definition $4 f$ has a weak sharp minima at $x^{\circ}$ if there exists a $\gamma>0$ and a $\delta>0$ such that

$$
f\left(x^{\circ}\right)+\gamma d\left(x, S_{f}\left(x^{\circ}\right)\right) \leq f(x)
$$

for all $x \in x^{\circ}+\delta B$.

It follows immediately from (19) that $f(x)=f\left(x^{\circ}\right)$ for any $x \in S_{f}\left(x^{\circ}\right) \cap\left(x^{\circ}+\right.$ $\delta B)$ in contrast to the definition of the local weak sharp minima in [6] where the set $S_{f}\left(x^{\circ}\right)$ must be the set of global minima of $f$.

Let $f$ be of the maximum type:

$$
f(x)=\max _{1 \leq i \leq n} f_{i}(x) .
$$

If each of the functions $f_{i}$ is defined by (18) and $x^{\circ} \in \cap_{i=1}^{n} \Omega_{i}$ then $f\left(x^{\circ}\right)=0$ and

$$
S_{f}\left(x^{\circ}\right)=\bigcap_{i=1}^{n} \Omega_{i} .
$$

It is easy to check that in this case (19) takes the form (15) with $\beta=\gamma^{-1}$. Thus, weak sharp minima for $f$ defined by $(20),(18)$ is equivalent to the metric inequality for the collection of sets $\Omega_{1}, \Omega_{2}, \ldots, \Omega_{n}$.

To establish the relation with the strong metric inequality and consequently with the strong regularity property of collections of sets considered in the current paper we need a stronger property than the one given by Definition 4 . This property is formulated in the next definition for functions of the maximum type (20). First denote

$$
\begin{gathered}
\tilde{f}\left(x ; x_{1}, \ldots, x_{n}\right)=\max _{1 \leq i \leq n} f_{i}\left(x+x_{i}\right), \\
\tilde{S}_{f}\left(x^{\circ} ; x_{1}, \ldots, x_{n}\right)=\left\{x \in X: \tilde{f}\left(x ; x_{1}, \ldots, x_{n}\right) \leq f\left(x^{\circ}\right)\right\} .
\end{gathered}
$$

Evidently

$$
\tilde{S}_{f}\left(x^{\circ} ; x_{1}, \ldots, x_{n}\right)=\bigcap_{i=1}^{n}\left\{x \in X: f_{i}\left(x+x_{i}\right) \leq f\left(x^{\circ}\right)\right\} .
$$

Definition 5 A function $f$ given by (20) has a relaxed sharp minima at $x^{\circ}$ if there exists a $\gamma>0$ and a $\delta>0$ such that

$$
f\left(x^{\circ}\right)+\gamma d\left(x, \tilde{S}_{f}\left(x^{\circ} ; x_{1}, \ldots, x_{n}\right)\right) \leq \tilde{f}\left(x ; x_{1}, \ldots, x_{n}\right)
$$

for all $x \in x^{\circ}+\delta B, x_{i} \in \delta B, i=1,2, \ldots, n$. 
In the case the functions $f_{i}$ are defined by (18) and $x^{\circ} \in \cap_{i=1}^{n} \Omega_{i}$ one has

$$
\tilde{S}_{f}\left(x^{\circ} ; x_{1}, \ldots, x_{n}\right)=\bigcap_{i=1}^{n}\left(\Omega_{i}-x_{i}\right)
$$

and (21) takes the form (14) with $\beta=\gamma^{-1}$. Thus, relaxed sharp minima for $f$ defined by (20), (18) appears equivalent to the strong metric inequality for the collection of sets $\Omega_{1}, \Omega_{2}, \ldots, \Omega_{n}$ and the following analog of Proposition 5 holds.

Proposition 6 The collection of sets $\Omega_{1}, \Omega_{2}, \ldots, \Omega_{n}$ is strongly regular at $x^{\circ}$ if and only if $f$ defined by (20), (18) has a relaxed sharp minima at $x^{\circ}$.

$\hat{\theta}\left[\Omega_{1}, \ldots, \Omega_{n}\right]\left(x^{\circ}\right)$ equals to the exact upper bound of all $\gamma$ from Definition 5.

\subsection{Multifunction criterion}

Another way of characterizing the regularity property of the collection of sets is through considering the corresponding property for some multifunction.

If $F: X \Rightarrow Y$ is a multifunction between normed spaces $X$ and $Y$ with the graph $\operatorname{gph} F=\{(x, y) \in X \times Y: y \in F(x)\}$ and $\left(x^{\circ}, y^{\circ}\right) \in \operatorname{gph} F$ one can introduce for it some analogs of $(1),(3),(5)$ (see $[15,16,18])$ :

$$
\begin{gathered}
\theta_{\rho}[F]\left(x^{\circ}, y^{\circ}\right)=\sup \left\{r \geq 0: y^{\circ}+r B \subset F\left(x^{\circ}+\rho B\right)\right\}, \\
\theta[F]\left(x^{\circ}, y^{\circ}\right)=\liminf _{\rho \rightarrow+0} \frac{\theta_{\rho}[F]\left(x^{\circ}, y^{\circ}\right)}{\rho}, \\
\hat{\theta}[F]\left(x^{\circ}, y^{\circ}\right)= \\
\liminf _{\substack {(x, y) \\
\begin{subarray}{c}{\operatorname{gph} F \\
\rho \rightarrow+0{ ( x , y ) \\
\begin{subarray} { c } { \operatorname { g p h } F \\
\rho \rightarrow + 0 } }\end{subarray}} \frac{\theta_{\rho}[F](x, y)}{\rho}
\end{gathered}
$$

and define the corresponding properties.

\section{Definition $6 F$ is}

(i) extremal at $\left(x^{\circ}, y^{\circ}\right)$ if $\theta_{\rho}[F]\left(x^{\circ}, y^{\circ}\right)=0$ for all $\rho>0$.

(ii) locally extremal at $\left(x^{\circ}, y^{\circ}\right)$ if $\theta_{\rho}[F]\left(x^{\circ}, y^{\circ}\right)=0$ for some $\rho>0$.

(iii) stationary at $\left(x^{\circ}, y^{\circ}\right)$ if $\theta[F]\left(x^{\circ}, y^{\circ}\right)=0$.

(iv) weakly stationary at $\left(x^{\circ}, y^{\circ}\right)$ if $\hat{\theta}[F]\left(x^{\circ}, y^{\circ}\right)=0$.

(v) regular at $\left(x^{\circ}, y^{\circ}\right)$ if $\theta[F]\left(x^{\circ}, y^{\circ}\right)>0$.

(vi) strongly regular at $\left(x^{\circ}, y^{\circ}\right)$ if $\hat{\theta}[F]\left(x^{\circ}, y^{\circ}\right)>0$.

The multifunction strong regularity condition can be reformulated as follows.

Proposition $7 F$ is strongly regular at $\left(x^{\circ}, y^{\circ}\right) \in \operatorname{gph} F$ if and only if there exists an $\alpha>0$ and $a \delta>0$ such that

$$
y+\alpha \rho B \subset F(x+\rho B)
$$

for all $\rho \in(0, \delta],(x, y) \in \operatorname{gph} F \cap\left(\left(x^{\circ}, y^{\circ}\right)+\delta B\right)$.

$\hat{\theta}[F]\left(x^{\circ}, y^{\circ}\right)$ equals to the exact upper bound of all such $\alpha$. 
(22) can be interpreted as the "inner" distance of $y^{\circ}$ in $F\left(x^{\circ}+\rho B\right)$ (the distance from $y^{\circ}$ to the complement of $F\left(x^{\circ}+\rho B\right)$ ). Taken with the negative sign it forms a part of the extended point-to-set "distance" function used e.g. in [12].

The regularity condition in Proposition 7 characterizes the covering property [9] (or linear openness) of $F$ near $\left(x^{\circ}, y^{\circ}\right)$. In the convex case this property was considered earlier by S. Robinson [30] when deriving the generalization of Banach open mapping theorem (see [11]). In general it is equivalent (see e.g. [25,31]) to the metric (or pseudo) regularity property [11,12] (and to the Aubin property [1, 31] of the inverse mapping). The metric regularity property can be considered as a kind of error bound condition for a multifunction (compare with Definition 3.3, part (b) from [26]).

All the extremality-stationarity-regularity concepts for multifunctions and for collections of sets are closely related. In particular, the following assertion holds, where as previously $\Omega_{1}, \Omega_{2}, \ldots, \Omega_{n}$ are subsets of $X$ and $x^{\circ} \in \cap_{i=1}^{n} \Omega_{i}$.

Proposition 8 Define $F: X \Rightarrow X^{n}: F(x)=\left(\Omega_{1}-x\right) \times\left(\Omega_{2}-x\right) \times \ldots \times\left(\Omega_{n}-x\right)$, $x \in X$. Then

(i) $\hat{\theta}\left[\Omega_{1}, \ldots \Omega_{n}\right]\left(x^{\circ}\right)=\hat{\theta}[F]\left(0, x^{\circ}, \ldots, x^{\circ}\right)$.

(ii) The collection of sets $\Omega_{1}, \Omega_{2}, \ldots, \Omega_{n}$ is strongly regular at $x^{\circ}$ if and only if $F$ is strongly regular at $\left(0, x^{\circ}, \ldots, x^{\circ}\right)$.

The proof of the first assertion of Proposition 8 in [19] (Theorem 3) was incomplete. For this reason the full proof is presented below.

Proof Due to the definition of $F$ conditions $\omega_{i} \in \Omega_{i}, i=1,2, \ldots, n$, are equivalent to the inclusion $\left(\omega_{1}, \ldots, \omega_{n}\right) \in F(0)$, and condition

$$
r B\left(\omega_{1}, \ldots, \omega_{n}\right) \subset F(\rho B)
$$

means that for any $a_{i} \in r B, i=1,2, \ldots, n$, there exists an $x \in \rho B$ such that $\omega_{i}+a_{i} \in$ $\Omega_{i}-x, i=1,2, \ldots, n$. This is equivalent to (9). Due to the definitions (2), (4), (5), (22)-(24) this implies the conditions

$$
\begin{gathered}
\theta_{\rho}\left[\Omega_{1}, \ldots \Omega_{n}\right]\left(\omega_{1}, \ldots, \omega_{n}\right)=\theta_{\rho}[F]\left(0, \omega_{1}, \ldots, \omega_{n}\right), \\
\theta\left[\Omega_{1}, \ldots \Omega_{n}\right]\left(\omega_{1}, \ldots, \omega_{n}\right)=\theta[F]\left(0, \omega_{1}, \ldots, \omega_{n}\right), \\
\hat{\theta}\left[\Omega_{1}, \ldots \Omega_{n}\right]\left(x^{\circ}\right) \geq \hat{\theta}[F]\left(0, x^{\circ}, \ldots, x^{\circ}\right) .
\end{gathered}
$$

The last condition is presented here as an inequality because when calculating the right-hand side of it one has to consider all sequences $\left(x, y_{1}, \ldots, y_{n}\right)$ from $\operatorname{gph} F$ converging to $\left(0, x^{\circ}, \ldots, x^{\circ}\right)$ (see (24)), not only those with $x=0$. However, the opposite inequality follows immediately if to notice that the inclusion $\left(x, y_{1}, \ldots, y_{n}\right) \in \operatorname{gph} F$ can be rewritten as $\left(0, x+y_{1}, \ldots, x+y_{n}\right) \in \operatorname{gph} F$. This proves the first assertion. The second one follows due to Definitions 1 and 6.

Another relation between regularity properties of multifunctions and collections of sets is given by the next statement from [19] (see Theorem 2 and Corollary 2.1).

Proposition 9 Let $F: X \Rightarrow Y$ and $\left(x^{\circ}, y^{\circ}\right) \in \operatorname{gph} F$. Define $\Omega_{1}=\operatorname{gph}(F), \Omega_{2}=$ $X \times\left\{y^{\circ}\right\}$. Then 
(i) $\hat{\theta}\left[\Omega_{1}, \Omega_{2}\right]\left(x^{\circ}, y^{\circ}\right) \leq \min \left(\hat{\theta}[F]\left(x^{\circ}, y^{\circ}\right) / 2,1\right) \leq 2 \hat{\theta}\left[\Omega_{1}, \Omega_{2}\right]\left(x^{\circ}, y^{\circ}\right)$.

(ii) $F$ is strongly regular at $\left(x^{\circ}, y^{\circ}\right)$ if and only if $\left\{\Omega_{1}, \Omega_{2}\right\}$ is strongly regular at $\left(x^{\circ}, y^{\circ}\right)$.

\subsection{Dual properties}

In this subsection the sets $\Omega_{1}, \Omega_{2}, \ldots, \Omega_{n}$ are assumed closed.

The dual stationarity/regularity properties are formulated in terms of Fréchet normal cones. Recall that the Fréchet normal cone to a set $\Omega$ at a point $x^{\circ} \in \Omega$ is defined as

$$
N\left(x^{\circ} \mid \Omega\right)=\left\{x^{*} \in X^{*}: \underset{x^{\Omega} \rightarrow x^{\circ}}{\limsup } \frac{\left\langle x^{*}, x-x^{\circ}\right\rangle}{\left\|x-x^{\circ}\right\|} \leq 0\right\} .
$$

Here $X^{*}$ is the space (topologically) dual to $X,\langle\cdot, \cdot\rangle$ is the bilinear form defining duality between $X$ and $X^{*}$ and $x \stackrel{\Omega}{\rightarrow} x^{\circ}$ means that $x \rightarrow x^{\circ}$ while $x \in \Omega$.

Using (25) it is possible to define one more constant for the collection of sets $\Omega_{1}, \Omega_{2}, \ldots, \Omega_{n}$ :

$$
\begin{aligned}
\eta\left[\Omega_{1}, \ldots, \Omega_{n}\right]\left(x^{\circ}\right)= & \lim _{\delta \rightarrow+0} \inf \left\{\left(\left\|\sum_{i=1}^{n} x_{i}^{*}\right\| / \sum_{i=1}^{n}\left\|x_{i}^{*}\right\|\right)_{\infty}:\right. \\
& \left.x_{i}^{*} \in N\left(x_{i} \mid \Omega_{i}\right), x_{i} \in \Omega_{i} \cap\left(x^{\circ}+\delta B\right), i=1, \ldots, n\right\} .
\end{aligned}
$$

Another "extended" division operation $(\cdot, \cdot)_{\infty}$ is used here. It differs from the $(\cdot, \cdot)_{\circ}$ operation used in (13), in the fourth rule definition:

4) $(0 / 0)_{\infty}=\infty$.

This allows one to exclude the case $x_{1}^{*}=x_{2}^{*}=\cdots=x_{n}^{*}=0$ when calculating (26). If this is the only case ( $x^{\circ} \in \operatorname{int} \cap_{i=1}^{n} \Omega_{i}$ ) one automatically gets $\eta\left[\Omega_{1}, \ldots, \Omega_{n}\right]\left(x^{\circ}\right)=\infty$.

Evidently constant (26) is nonnegative. It can be used for defining (dual) stationarity/regularity properties.

Definition 7 The collection of sets $\Omega_{1}, \Omega_{2}, \ldots, \Omega_{n}$ is

(i) $\eta$-stationary at $x^{\circ}$ if $\eta\left[\Omega_{1}, \ldots, \Omega_{n}\right]\left(x^{\circ}\right)=0$;

(ii) $\eta$-regular at $x^{\circ}$ if $\eta\left[\Omega_{1}, \ldots, \Omega_{n}\right]\left(x^{\circ}\right)>0$.

The next proposition gives equivalent characterization of $\eta$-stationarity and $\eta$-regularity in terms of normal elements. It follows directly from (26).

Proposition 10 The collection of sets $\Omega_{1}, \Omega_{2}, \ldots, \Omega_{n}$ is

(i) $\eta$-stationary at $x^{\circ}$ if and only if for any $\delta>0$ there exist $x_{i} \in \Omega_{i} \cap\left(x^{\circ}+\delta B\right)$, $x_{i}^{*} \in N\left(x_{i} \mid \Omega_{i}\right), i=1, \ldots, n$, such that

$$
\left\|\sum_{i=1}^{n} x_{i}^{*}\right\|<\delta \quad \sum_{i=1}^{n}\left\|x_{i}^{*}\right\|=1
$$


(ii) $\eta$-regular at $x^{\circ}$ if and only if there exists $a \gamma>0$ and $a \delta>0$ such that

$$
\left\|\sum_{i=1}^{n} x_{i}^{*}\right\| \geq \gamma \sum_{i=1}^{n}\left\|x_{i}^{*}\right\|
$$

for all $x_{i}^{*} \in N\left(x_{i} \mid \Omega_{i}\right), x_{i} \in \Omega_{i} \cap\left(x^{\circ}+\delta B\right), i=1, \ldots, n$. $\eta\left[\Omega_{1}, \ldots, \Omega_{n}\right]\left(x^{\circ}\right)$ equals to the exact upper bound of all such $\gamma$.

Note that (27) constitutes the "fuzzy" nonconvex separation property for the collection of sets: the collection of dual space elements $x_{i}^{*}, i=1, \ldots, n$, separates the sets "up to $\delta$ ". In view of Proposition $10 \eta$-stationarity can be referred to as normal separability.

The next theorem gives the relations between (26) and (5).

\section{Theorem 2 ([18])}

(i) $\hat{\theta}\left[\Omega_{1}, \ldots, \Omega_{n}\right]\left(x^{\circ}\right) \leq \eta\left[\Omega_{1}, \ldots, \Omega_{n}\right]\left(x^{\circ}\right)$.

(ii) If $X$ is Asplund and $\hat{\theta}\left[\Omega_{1}, \ldots, \Omega_{n}\right]\left(x^{\circ}\right)<1$ then

$$
\eta\left[\Omega_{1}, \ldots, \Omega_{n}\right]\left(x^{\circ}\right) \leq \frac{\hat{\theta}\left[\Omega_{1}, \ldots, \Omega_{n}\right]\left(x^{\circ}\right)}{1-\hat{\theta}\left[\Omega_{1}, \ldots, \Omega_{n}\right]\left(x^{\circ}\right)} .
$$

It follows from the first part of Theorem 2 that strong regularity of a collection of sets implies its $\eta$-regularity, while the second part asserts equivalence of the two types of regularity in the Asplund space environment.

Corollary 2 Let $X$ be Asplund. The collection of sets $\Omega_{1}, \Omega_{2}, \ldots, \Omega_{n}$ is strongly regular at $x^{\circ}$ if and only if it is $\eta$-regular at $x^{\circ}$.

The last statement can, of course, be reformulated as equivalence of the two types of stationarity (Extended extremal principle [17]). Taking into account the extremal characterizations of Asplund spaces in [24] one can conclude that asplundity of the space is not only sufficient but also necessary for the Extended extremal principle to be valid (see [17]). This gives another proof of the well known fact that, being a rather rich subclass of general Banach spaces (see [28]), Asplund spaces provide the appropriate framework for using Fréchet normals and subdifferentials.

Definition (26) can be simplified if one makes use of the strict $\delta$-normal cone $[13,17](\delta \geq 0)$ to a closed set $\Omega$ at $x^{\circ} \in \Omega$ :

$$
\hat{N}_{\delta}\left(x^{\circ} \mid \Omega\right)=\bigcup_{x \in \Omega \cap\left(x^{\circ}+\delta B\right)} N(x \mid \Omega) .
$$

Note that this cone can be nonconvex.

Using (30) one can rewrite (26) as

$$
\begin{aligned}
\eta\left[\Omega_{1}, \ldots, \Omega_{n}\right]\left(x^{\circ}\right)=\lim _{\delta \rightarrow+0} \inf \left\{\left(\left\|\sum_{i=1}^{n} x_{i}^{*}\right\| / \sum_{i=1}^{n}\left\|x_{i}^{*}\right\|\right)_{\infty}:\right. \\
\left.x_{i}^{*} \in \hat{N}_{\delta}\left(x^{\circ} \mid \Omega_{i}\right), i=1, \ldots, n,\right\}
\end{aligned}
$$


and assertion (ii) in Proposition 10 can be replaced by the following (equivalent) one.

(ii') There exists a $\gamma>0$ and a $\delta>0$ such that (28) holds for all $x_{i}^{*} \in N_{\delta}\left(x^{\circ} \mid \Omega_{i}\right)$, $i=1, \ldots, n$.

In its turn, the last condition can be rewritten equivalently as

(SG) There exists an $\alpha>0$ and a $\delta>0$ such that for any $x^{*} \in \sum_{i=1}^{n} N_{\delta}\left(x^{\circ} \mid \Omega_{i}\right)$ the next inequality holds:

$$
\sup \left\{\sum_{i=1}^{n}\left\|x_{i}^{*}\right\|: x_{i}^{*} \in N_{\delta}\left(x^{\circ} \mid \Omega_{i}\right), i=1, \ldots, n, \sum_{i=1}^{n} x_{i}^{*}=x^{*}\right\} \leq \alpha\left\|x^{*}\right\| .
$$

It implies another regularity condition:

(G) There exists an $\alpha>0$ and a $\delta>0$ such that for any $x^{*} \in \sum_{i=1}^{n} N_{\delta}\left(x^{\circ} \mid \Omega_{i}\right)$ the next inequality holds:

$$
\inf \left\{\sum_{i=1}^{n}\left\|x_{i}^{*}\right\|: x_{i}^{*} \in N_{\delta}\left(x^{\circ} \mid \Omega_{i}\right), i=1, \ldots, n, \sum_{i=1}^{n} x_{i}^{*}=x^{*}\right\} \leq \alpha\left\|x^{*}\right\| .
$$

The reverse implication $(\mathrm{G}) \Rightarrow(\mathrm{SG})$ does not hold in general even for convex sets: consider the example at the end of subsection 3.3.

Note that condition $(\mathrm{G})$ is actually Jameson's property $(G)$ (see $[3,26]$ ) for the collection of strict $\delta$-normal cones. If to adopt this terminology, condition $(\mathrm{SG})$ can be addressed to as the strong property $(G)$. Thus, strong regularity of the collection of sets is equivalent to the strong property $(\mathrm{G})$.

It is easy to see that (SG) implies also strong additive regularity [7] of the collection of strict $\delta$-normal cones.

\section{Examples}

This section is devoted to considering examples of strongly regular collections of sets which can be important for applications.

\subsection{Strengthening condition (a)}

The main idea developed in this subsection is to provide meaningful sufficient conditions for the condition (a) of Proposition 2 to be satisfied. The simplest condition of this kind is given by Corollary 1 .

It follows from Proposition 2 that for the collection of sets $\Omega_{1}, \Omega_{2}, \ldots, \Omega_{n}$ to be strongly regular at $x^{\circ}$, the intersection of the sets $\Omega_{i}-\omega_{i}$ must be sufficiently rich for all $\omega_{i} \in \Omega_{i}$ near $x^{\circ}, i=1,2, \ldots, n$.

Proposition 11 Consider the following conditions for the collection of sets $\Omega_{1}$, $\Omega_{2}, \ldots, \Omega_{n}$ near $x^{\circ}$ : 
(b) There exists an $\alpha>0$ and $a \delta>0$ such that for any $\omega_{i} \in \Omega_{i} \cap\left(x^{\circ}+\delta B\right)$, $i=1,2, \ldots, n, \rho \in(0, \delta]$, and any $a \in \alpha \rho B$ one can find an $x \in \rho B$ such that

$$
x+\alpha \rho B \subset \bigcap_{i=1}^{n-1}\left(\Omega_{i}-\omega_{i}\right), \quad x+a \in \Omega_{n}-\omega_{n} .
$$

(c) There exists an $\alpha>0$ and $a \delta>0$ such that for any $\omega_{i} \in \Omega_{i} \cap\left(x^{\circ}+\delta B\right)$, $i=1,2, \ldots, n$, and any $a \in \alpha B$ there exists $a c \in X$ with $\|c\|=1$ such that for any $\rho \in(0, \delta]$ one can find $a t \in(0,1]$ such that

$$
\rho(t c+\alpha B) \subset \bigcap_{i=1}^{n-1}\left(\Omega_{i}-\omega_{i}\right), \quad \rho(t c+a) \in \Omega_{n}-\omega_{n} .
$$

(d) There exists an $\alpha>0$ and $a \delta>0$ such that for any $\omega_{i} \in \Omega_{i} \cap\left(x^{\circ}+\delta B\right)$, $i=1,2, \ldots, n$, and any $a \in \alpha B$ there exists $a c \in X$ with $\|c\|=1$ such that

$$
\text { cone }(c+\alpha B) \cap \delta B \subset \bigcap_{i=1}^{n-1}\left(\Omega_{i}-\omega_{i}\right), \quad \operatorname{cone}\{c+a\} \cap(\delta B) \subset \Omega_{n}-\omega_{n} .
$$

(e) There exists an $\alpha>0, a \delta>0$ and a cone $C$ such that for any $\omega_{i} \in \Omega_{i} \cap\left(x^{\circ}+\right.$ $\delta B), i=1,2, \ldots, n$, one has $\omega_{i}+C \subset \Omega_{i}, i=1,2, \ldots, n-1$, and for any $a \in \alpha B$ there exists a $c \in X$ with $\|c\|=1$ such that

$$
c+\alpha B \subset C, \quad \text { cone }\{c+a\} \subset \Omega_{n}-\omega_{n} .
$$

The following assertions hold true:

(i) $(e) \Rightarrow(d) \Rightarrow(c) \Rightarrow(b) \Rightarrow(a)$.

(ii) If any of the conditions $(b),(c),(d),(e)$ is satisfied then

- $\hat{\theta}\left[\Omega_{1}, \ldots, \Omega_{n}\right]\left(x^{\circ}\right) \geq \alpha$;

- the collection of sets $\Omega_{1}, \Omega_{2}, \ldots, \Omega_{n}$ is strongly regular at $x^{\circ}$.

The notation cone $\Omega$ appeared in (d) denotes the cone spanned on $\Omega$ :

$$
\text { cone } \Omega=\{t \omega: t \geq 0, \omega \in \Omega\} \text {. }
$$

Proof (i). (b) $\Rightarrow$ (a). Take arbitrary $a_{i} \in \alpha \rho B, i=1,2, \ldots, n$. It follows from (b) that $x+a_{i} \in \Omega_{i}-\omega_{i}, i=1,2, \ldots, n$, for some $x \in \rho B$ and consequently

$$
x \in \bigcap_{i=1}^{n}\left(\Omega_{i}-\omega_{i}-a_{i}\right)
$$

Since $x \in \rho B$ the last inclusion implies (9). The assertion follows from Proposition 2.

(c) $\Rightarrow$ (b). Let $\alpha>0$ and a $\delta>0$ satisfying (c) be given and take any $\rho \in(0, \delta]$, $\omega_{i} \in \Omega_{i} \cap\left(x^{\circ}+\delta B\right), i=1,2, \ldots, n$, and any $a \in \alpha \rho B$. Denote $b=a / \rho$. Then $b \in \alpha B$ and it follows from (c) that there exists a $c \in X$ with $\|c\|=1$ and a $t \in(0,1]$ such that (32) holds true (with $b$ instead of $a$ ). Denote $x=t \rho c$. Then $x \in \rho B$ and (31) holds true. 
(d) $\Rightarrow$ (c). Let $\alpha>0$ and a $\delta>0$ satisfying (d) be given. Take $\alpha^{\prime}=\min (\alpha, 1)$, $\delta^{\prime}=\delta / 2$. Then for any $c \in X, \rho \in[0, \delta]$ one evidently has $\rho\left(c+\alpha^{\prime} B\right) \subset$ cone $(c+$ $\alpha B)$, and the condition $x \in \rho\left(c+\alpha^{\prime} B\right)$ implies $\|x\| \leq \delta^{\prime}\left(1+\alpha^{\prime}\right) \leq \delta$. Thus

$$
\rho\left(c+\alpha^{\prime} B\right) \subset \text { cone }(c+\alpha B) \cap(\delta B) .
$$

If $\omega_{i} \in \Omega_{i} \cap\left(x^{\circ}+\delta^{\prime} B\right), i=1,2, \ldots, n$, and $a \in \alpha^{\prime} B$ then (33) holds true for some $c \in X$ with $\|c\|=1$. These conditions together with (34) imply (c) with $t=1$.

(e) $\Rightarrow(d)$. This implication follows from the inclusions

$$
c+\alpha B \subset C \subset \bigcap_{i=1}^{n-1}\left(\Omega_{i}-\omega_{i}\right) .
$$

(ii). Since in all the cases considered above $\alpha$ satisfies (a), the second assertion is an immediate corollary of the first one due to Proposition 2.

Condition (b) certainly implies the traditional for the convex case regularity condition

$$
\bigcap_{i=1}^{n-1} \operatorname{int} \Omega_{i} \bigcap \Omega_{n} \neq \emptyset \text {. }
$$

Actually it is the necessity to cover the convex case that led to treating one of the sets separately in all the conditions in Proposition 11. Note that not all the sets need to have nonempty interiors. Of course, conditions (b)-(c) can be replaced by the stronger ones with all the sets treated similarly:

$\left(\mathrm{b}^{\prime}\right)$ There exists an $\alpha>0$ and a $\delta>0$ such that for any $\omega_{i} \in \Omega_{i} \cap\left(x^{\circ}+\delta B\right)$, $i=1,2, \ldots, n, \rho \in(0, \delta]$, one can find an $x \in \rho B$ such that

$$
x+\alpha \rho B \subset \bigcap_{i=1}^{n}\left(\Omega_{i}-\omega_{i}\right) .
$$

( $\left.\mathrm{c}^{\prime}\right)$ There exists an $\alpha>0$ and a $\delta>0$ such that for any $\omega_{i} \in \Omega_{i} \cap\left(x^{\circ}+\delta B\right)$, $i=1,2, \ldots, n$, there exists a $c \in X$ with $\|c\|=1$ such that for any $\rho \in(0, \delta]$ one can find a $t \in(0,1]$ such that

$$
\rho(t c+\alpha B) \subset \bigcap_{i=1}^{n}\left(\Omega_{i}-\omega_{i}\right) .
$$

$\left(\mathrm{d}^{\prime}\right)$ There exists an $\alpha>0$ and a $\delta>0$ such that for any $\omega_{i} \in \Omega_{i} \cap\left(x^{\circ}+\delta B\right)$, $i=1,2, \ldots, n$, there exists a $c \in X$ with $\|c\|=1$ such that

$$
\text { cone }(c+\alpha B) \cap(\delta B) \subset \bigcap_{i=1}^{n}\left(\Omega_{i}-\omega_{i}\right) .
$$

( $\left.\mathrm{e}^{\prime}\right)$ There exists an $\alpha>0$, a $\delta>0$ and a cone $C$ such that $c+\alpha B \subset C$ for some $c \in X$ with $\|c\|=1$, and $\omega_{i}+C \subset \Omega_{i}$ for any $\omega_{i} \in \Omega_{i} \cap\left(x^{\circ}+\delta B\right)$, $i=1,2, \ldots, n$. 
Condition ( $\left.\mathrm{b}^{\prime}\right)$ basically states that $\cap_{i=1}^{n}\left(\Omega_{i}-\omega_{i}\right)$ contains a sequence of balls $x_{k}+r_{k} B, k=1,2, \ldots$, such that $x_{k} \rightarrow 0$ and $r_{k} \geq \alpha\left\|x_{k}\right\|$ for some $\alpha>0$. Condition $\left(c^{\prime}\right)$ strengthens $\left(b^{\prime}\right)$ further by assuming that all the ball centers lie on the same ray. Condition $\left(\mathrm{d}^{\prime}\right)$ actually corresponds to fixing $t=1$ in $\left(\mathrm{c}^{\prime}\right)$.

If to consider $C$ in $\left(\mathrm{e}^{\prime}\right)$ as a cone of nonpositive elements in $X$ then $\left(\mathrm{e}^{\prime}\right)$ means that all the sets $\Omega_{i}, i=1,2, \ldots, n$, are locally downward (see [32]) with respect to $C$ near $x^{\circ} . C$ is not assumed to be convex and in general does not define any pre-order in $X$. However, one can always take $C=$ cone $(c+\alpha B)$.

It is well known that collections of downward sets are strongly regular at any common point. The metric inequality (15) holds true for them as an equality with $\beta=1$ (see [32]).

Condition $\left(\mathrm{d}^{\prime}\right)$ can also be viewed as a local version of the downward property with the cone cone $(c+\alpha B)$ considered locally and depending on the choice of $\omega_{i} \in \Omega_{i} \cap\left(x^{\circ}+\delta B\right), i=1,2, \ldots, n$.

\subsection{Functional conditions}

In this subsection we consider the case when the sets are defined in terms of some (set-valued) functions. More specifically, let

$$
\Omega_{i}=\left\{x \in X: F_{i}(x) \cap C_{i} \neq \emptyset\right\}, i=1,2, \ldots, n,
$$

where $F_{i}$ is a set-valued mapping (multifunction) from $X$ into a normed space $Y_{i}$ and $C_{i}$ is a nonempty subset in $Y_{i}$. We still assume $\Omega_{i}$ to be closed, as is the case, for example, when $C_{i}$ is closed and $F_{i}$ is a continuous function.

Proposition 2 in this case takes the following form.

Proposition 12 The collection of sets (35) is strongly regular at $x^{\circ}$ if and only if there exists an $\alpha>0$ and $a \delta>0$ such that for any $\rho \in(0, \delta], x_{i} \in x^{\circ}+\delta B$ with $F_{i}\left(x_{i}\right) \cap C_{i} \neq \emptyset, i=1,2, \ldots, n$, and any $a_{i} \in \alpha \rho B$ one can find an $x \in \rho B$ such that $F_{i}\left(x+x_{i}+a_{i}\right) \cap C_{i} \neq \emptyset, i=1,2, \ldots, n$.

If to consider (35) as a constrained system then Proposition 12 gives a kind of constraint qualification. It takes a more familiar form if one imposes additional assumptions on the parameters.

Let $Y_{i}=\mathbb{R}, F_{i}$ be a continuous (single-valued) function and let $C_{i}=\mathbb{R}_{-}$, $i=1,2, \ldots, m(m \leq n), C_{i}=\{0\}, i=m+1, \ldots, n$. Then (35) takes the form of a system of inequalities and equalities:

$$
\begin{gathered}
\Omega_{i}=\left\{x \in X: F_{i}(x) \leq 0\right\}, i=1,2, \ldots, m, \\
\Omega_{i}=\left\{x \in X: F_{i}(x)=0\right\}, i=m+1, \ldots, n .
\end{gathered}
$$

Denote by $I$ the set of active constraints: $I=\left\{i \in\{1,2, \ldots, m\}: F_{i}\left(x^{\circ}\right)=0\right\}$. Proposition 12 can be rewritten as follows:

Proposition 13 The collection of sets (36), (37) is strongly regular at $x^{\circ}$ if and only if there exists an $\alpha>0$ and $a \delta>0$ such that for any $\rho \in(0, \delta], x_{i} \in x^{\circ}+\delta B$ with

$$
\begin{gathered}
F_{i}\left(x_{i}\right) \leq 0, i \in I, \\
F_{i}\left(x_{i}\right)=0, i=m+1, \ldots, n,
\end{gathered}
$$


and any $a_{i} \in \alpha \rho B$ one can find an $x \in \rho B$ such that

$$
\begin{gathered}
F_{i}\left(x+x_{i}+a_{i}\right) \leq 0, i \in I, \\
F_{i}\left(x+x_{i}+a_{i}\right)=0, i=m+1, \ldots, n .
\end{gathered}
$$

Proposition 13 contains traditional primal space constraint qualifications for nonlinear programming problems. This can be illustrated by the next Proposition covering the convex case with inequality type constraints. The differentiable case with equality type constraints will be considered elsewhere.

Proposition 14 Let $F_{i}: X \rightarrow \mathbb{R}, i=1,2, \ldots, m$, be convex. The collection of sets (36) is strongly regular at $x^{\circ}$ provided that the Slater condition is satisfied:

(S) There exists a $z \in X$ such that $F_{i}(z)<0, i \in I$.

Proof Let us show that the Slater condition implies the conditions formulated in Proposition 13 for the case when only inequality-type constraints are present. If $(\mathrm{S})$ is valid then due to the continuity of $F_{i}: X \rightarrow \mathbb{R}, i \in I$, one can find a $\delta>0$ such that $F_{i}(u) \leq 0$ for all $u \in z+2 \delta B$ and all $i \in I$. Take arbitrary $\rho \in(0, \delta]$, $x_{i} \in x^{\circ}+\delta B$ with $F_{i}\left(x_{i}\right) \leq 0, a_{i} \in \delta \rho B$ and consider $x=\rho\left(z-x^{\circ}\right)$. Then

$$
F_{i}\left(x+x_{i}+a_{i}\right)=F_{i}\left(\rho\left(z+x_{i}-x^{\circ}+a_{i} / \rho\right)+(1-\rho) x_{i}\right) \leq \rho F_{i}\left(z+x_{i}-x^{\circ}+a_{i} / \rho\right)
$$

due to convexity of $F_{i}$. Obviously, $\left\|x_{i}-x^{\circ}+a_{i} / \rho\right\| \leq 2 \delta$ and consequently $F_{i}(x+$ $\left.x_{i}+a_{i}\right) \leq 0$. Thus, the conditions of Proposition 13 are satisfied (with $\alpha=\delta$ ).

Some additional properties of sets of type (35) can be established in the case when they are defined with the help of strongly regular multifunctions. The next proposition follows directly from Proposition 7.

Proposition 15 Let

$$
\Omega=\{x \in X: F(x) \cap C \neq \emptyset\},
$$

where $F: X \Rightarrow Y$ is strongly regular at $\left(x^{\circ}, y^{\circ}\right) \in \operatorname{gph} F$ with $y^{\circ} \in C$. Then there exists an $\alpha>0$ and $a \delta>0$ such that for any $\rho \in(0, \delta], x \in \Omega \cap\left(x^{\circ}+\delta B\right)$, $y \in F(x) \cap\left(y^{\circ}+\delta B\right), v \in y+\alpha \rho B$ there exists $a u \in F^{-1}(v) \cap(x+\rho B)$.

When $v$ is limited to $C$ Proposition 15 gives some regularity conditions for the set $\Omega$ : it guarantees that $\Omega$ is sufficiently rich near $x^{\circ}$. On the other hand, when $v \notin C$ it gives some error bounds. Examples of conditions of these types are given by the next proposition which is actually a corollary of Proposition 15 .

Proposition 16 Let $\Omega$ be defined by (38), where $F=\left(F_{1}, F_{2}, \ldots, F_{n}\right): X \rightarrow \mathbb{R}^{n}$, $C=\mathbb{R}^{m} \times 0_{n-m}, y^{\circ}=F\left(x^{\circ}\right) \in C$, and let $F$ be strongly regular at $\left(x^{\circ}, y^{\circ}\right)$. Then there exists an $\alpha>0$ and $a \delta>0$ such that for any $\rho \in(0, \delta], x \in x^{\circ}+\delta B$ with $F(x) \in C \cap\left(y^{\circ}+\delta B\right)$ one can find

(i) $a u_{1} \in x+\rho B$ such that

$$
\begin{gathered}
F_{i}\left(u_{1}\right) \leq F_{i}(x)-\alpha \rho, i=1,2, \ldots, m, \\
F_{i}\left(u_{1}\right)=0, i=m+1, \ldots, n,
\end{gathered}
$$


(ii) $a u_{2} \in x+\rho B$ such that

$$
\begin{gathered}
F_{i}\left(u_{2}\right) \geq F_{i}(x)+\alpha \rho, i=1,2, \ldots, m, \\
\left|F_{i}\left(u_{2}\right)\right| \geq \alpha \rho, i=m+1, \ldots, n .
\end{gathered}
$$

Certainly it can make sense to consider a weakened version of Proposition 16 if to assume strong regularity not of the "whole" of $F$, but of the mapping consisting only of those components which correspond to equalities and active inequalities. The necessary changes in the conclusion part of the statement are obvious.

Acknowledgements I wish to thank Alexander Rubinov for attracting my attention to downward sets and other important for applications examples of strongly regular collections of sets considered in Section 4 as well as for numerous helpful discussions; other colleagues who read the preliminary version of the paper and provided me with their comments, especially Bernd Kummer for noticing the similarity of (22) and the extended point-to-set distance function, and an anonymous colleague for attracting my attention to the concept of weak sharp minima and providing me with the references [6-8]; anonymous referees for careful reading of the paper and many valuable comments, especially for pointing out a gap in one of the proofs which led to changes in some definitions and for attracting my attention to the work by S. Robinson [30].

\section{References}

1. Aubin, J.P., Ekeland, I.: Applied Nonlinear Analysis. John Wiley, New York (1984)

2. Bakan, A., Deutsch, F., Li, W.: Strong chip, normality, and linear regularity of convex sets. Trans. Amer. Math. Soc. 357(10), 3831-3863 (2005)

3. Bauschke, H.H., Borwein, J.M., Li, W.: Strong conical hull intersection property, bounded linear regularity, Jameson's property $(G)$, and error bounds in convex optimization. Math. Program., Ser. A 86(1), 135-160 (1999)

4. Bauschke, H.H., Borwein, J.M., Tseng, P.: Bounded linear regularity, strong CHIP, and CHIP are distinct properties. J. Convex Anal. 7(2), 395-412 (2000)

5. Borwein, J.M., Jofré, A.: A nonconvex separation property in Banach spaces. Math. Meth. Oper. Res. 48, 169-179 (1998)

6. Burke, J.V., Deng, S.: Weak sharp minima revisited. I. Basic theory. Control Cybernet. 31(3), 439-469 (2002). Well-posedness in optimization and related topics (Warsaw, 2001)

7. Burke, J.V., Deng, S.: Weak sharp minima revisited. II. Application to linear regularity and error bounds. Math. Program., Ser. B 104(2-3), 235-261 (2005)

8. Burke, J.V., Ferris, M.C.: Weak sharp minima in mathematical programming. SIAM J. Control Optim. 31(5), 1340-1359 (1993)

9. Dmitruk, A.V., Milyutin, A.A., Osmolovsky, N.P.: Lyusternik's theorem and the theory of extrema. Russian Math. Surveys 35, 11-51 (1980)

10. Dubovitskii, A.Y., Milyutin, A.A.: Extremum problems in the presence of restrictions. U.S.S.R. Comp. Maths. Math. Phys. 5, 1-80 (1965)

11. Ioffe, A.D.: Metric regularity and subdifferential calculus. Russian Math. Surveys 55, 501$558(2000)$

12. Klatte, D., Kummer, B.: Nonsmooth Equations in Optimization: Regularity, Calculus, Methods and Applications, Nonconvex Optimization and Its Applications, vol. 60. Kluwer Academic Publishers, Dordrecht (2002)

13. Kruger, A.Y.: On calculus of strict $\varepsilon$-semidifferentials. Dokl. Akad. Nauk Belarusi 40(4), 34-39 (1996). In Russian

14. Kruger, A.Y.: On extremality of set systems. Dokl. Nats. Akad. Nauk Belarusi 42(1), 24-28 (1998). In Russian

15. Kruger, A.Y.: Strict $(\varepsilon, \delta)$-semidifferentials and extremality of sets and functions. Dokl. Nats. Akad. Nauk Belarusi 44(4), 21-24 (2000). In Russian

16. Kruger, A.Y.: Strict $(\varepsilon, \delta)$-semidifferentials and extremality conditions. Optimization 51, 539-554 (2002) 
17. Kruger, A.Y.: On Fréchet subdifferentials. J. Math. Sci. (N. Y.) 116(3), 3325-3358 (2003). Optimization and related topics, 3

18. Kruger, A.Y.: Weak stationarity: eliminating the gap between necessary and sufficient conditions. Optimization 53, 147-164 (2004)

19. Kruger, A.Y.: Stationarity and regularity of set systems. Pac. J. Optim. 1(1), 101-126 (2005)

20. Kruger, A.Y.: Stationarity and regularity of real-valued functions. Appl. Comput. Math. (2006). To appear

21. Kruger, A.Y., Mordukhovich, B.S.: Extremal points and the Euler equation in nonsmooth optimization. Dokl. Akad. Nauk BSSR 24(8), 684-687 (1980). In Russian

22. Mordukhovich, B.S.: The extremal principle and its applications to optimization and economics. In: A. Rubinov, B. Glover (eds.) Optimization and Related Topics, Applied Optimization, Vol. 47, pp. 343-369. Kluwer Academic Publishers, Dordrecht (2001)

23. Mordukhovich, B.S.: Variational Analysis and Generalized Differentiation, I: Basic Theory, II: Applications, Grundlehren Series (Fundamental Principles of Mathematical Sciences), vol. 330, 331. Springer-Verlag, New York (2005)

24. Mordukhovich, B.S., Shao, Y.: Extremal characterizations of Asplund spaces. Proc. Amer. Math. Soc. 124, 197-205 (1996)

25. Mordukhovich, B.S., Shao, Y.H.: Differential characterizations of covering, metric regularity, and Lipschitzian properties of multifunctions between Banach spaces. Nonlinear Anal. 25(12), 1401-1424 (1995)

26. Ng, K.F., Yang, W.H.: Regularities and their relations to error bounds. Math. Program., Ser A 99, 521-538 (2004)

27. Ngai, H.V., Théra, M.: Metric inequality, subdifferential calculus and applications. SetValued Anal. 9, 187-216 (2001)

28. Phelps, R.R.: Convex Functions, Monotone Operators and Differentiability, 2nd edition. Lecture Notes in Mathematics, Vol. 1364. Springer-Verlag, New York (1993)

29. Polyak, B.T.: Introduction to Optimization. Translations Series in Mathematics and Engineering. Optimization Software Inc. Publications Division, New York (1987). Translated from Russian

30. Robinson, S.M.: Regularity and stability for convex multivalued functions. Math. Oper. Res. 1(2), 130-143 (1976)

31. Rockafellar, R.T., Wets, R.J.B.: Variational Analysis. Springer-Verlag, New York (1998)

32. Rubinov, A.M.: Abstract Convexity and Global Optimization. Kluwer Academic Publishers, Dordrecht (2000) 